\title{
Electron transport in acetate-grown Methanosarcina acetivorans
}

Mingyu Wang ${ }^{1}$, Jean-Francois Tomb ${ }^{2}$ and James G Ferry ${ }^{1 *}$

\begin{abstract}
Background: Acetate is the major source of methane in nature. The majority of investigations have focused on acetotrophic methanogens for which energy-conserving electron transport is dependent on the production and consumption of $\mathrm{H}_{2}$ as an intermediate, although the great majority of acetotrophs are unable to metabolize $\mathrm{H}_{2}$. The presence of cytochrome $\mathrm{c}$ and a complex (Ma-Rnf) homologous to the Rnf ( $\underline{R}$ hodobacter $\underline{\boldsymbol{n}}$ itrogen $\boldsymbol{f} \mathbf{i x a t i o n})$ complexes distributed in the domain Bacteria distinguishes non- $\mathrm{H}_{2}$-utilizing Methanosarcina acetivorans from $\mathrm{H}_{2}-$ utilizing species suggesting fundamentally different electron transport pathways. Thus, the membrane-bound electron transport chain of acetate-grown $M$. acetivorans was investigated to advance a more complete understanding of acetotrophic methanogens.

Results: A component of the CO dehydrogenase/acetyl-CoA synthase (CdhAE) was partially purified and shown to reduce a ferredoxin purified using an assay coupling reduction of the ferredoxin to oxidation of CdhAE. Mass spectrometry analysis of the ferredoxin identified the encoding gene among annotations for nine ferredoxins encoded in the genome. Reduction of purified membranes from acetate-grown cells with ferredoxin lead to reduction of membrane-associated multi-heme cytochrome $c$ that was re-oxidized by the addition of either the heterodisulfide of coenzyme M and coenzyme B (CoM-S-S-CoB) or 2-hydoxyphenazine, the soluble analog of methanophenazine (MP). Reduced 2-hydoxyphenazine was re-oxidized by membranes that was dependent on addition of CoM-S-S-CoB. A genomic analysis of Methanosarcina thermophila, a non- $\mathrm{H}_{2}$-utilizing acetotrophic methanogen, identified genes homologous to cytochrome $c$ and the Ma-Rnf complex of M. acetivorans.

Conclusions: The results support roles for ferredoxin, cytochrome $c$ and MP in the energy-conserving electron transport pathway of non- $\mathrm{H}_{2}$-utilizing acetotrophic methanogens. This is the first report of involvement of a cytochrome $\mathrm{c}$ in acetotrophic methanogenesis. The results suggest that diverse acetotrophic Methanosarcina species have evolved diverse membrane-bound electron transport pathways leading from ferredoxin and culminating with MP donating electrons to the heterodisulfide reductase (HdrDE) for reduction of CoM-S-S-CoB.
\end{abstract}

\section{Background}

The decomposition of complex organic matter to methane (biomethanation) in diverse anaerobic habitats of Earth's biosphere involves an anaerobic microbial food chain comprised of distinct metabolic groups, the first of which metabolizes the complex organic matter primarily to acetate and also formate or $\mathrm{H}_{2}$ that are growth substrates for two distinct methane-producing groups (methanogens) [1]. The methyl group of acetate

\footnotetext{
* Correspondence: jgf3@psu.edu

'Department of Biochemistry and Molecular Biology, Eberly College of Science, The Pennsylvania State University, University Park, Pennsylvania 16802-4500, USA

Full list of author information is available at the end of the article
}

contributes most of the methane produced in the biomethanation process via the aceticlastic pathway whereas the remainder originates primarily from the reduction of $\mathrm{CO}_{2}$ with electrons derived from the oxidation of formate or $\mathrm{H}_{2}$ in the $\mathrm{CO}_{2}$-reduction pathway $[2,3]$. Smaller, albeit significant, amounts of methane derive from the methyl groups of methanol, methylamines and dimethylsulfide [1].

Only two genera of aceticlastic methanogens have been described, Methanosarcina and Methanosaeta [2]. In both genera, the $\mathrm{CO}$ dehydrogenase/acetyl-CoA complex (Cdh) cleaves activated acetate into methyl and carbonyl groups. The methyl group is transferred to coenzyme $\mathrm{M}$ (HS-CoM) producing $\mathrm{CH}_{3}-\mathrm{S}-\mathrm{CoM}$ that is
C Biomed Central

() 2011 Wang et al; licensee BioMed Central Ltd. This is an Open Access article distributed under the terms of the Creative Commons Attribution License (http://creativecommons.org/licenses/by/2.0), which permits unrestricted use, distribution, and reproduction in any medium, provided the original work is properly cited. 
reductively demethylated to methane with electrons donated by coenzyme B (HS-CoB). The heterodisulfide CoM-S-S-CoB is a product of the demethylation reaction that is reduced to the sulfhydryl forms of the cofactors by heterodisulfide reductase ( $\mathrm{Hdr})$. The proton gradient driving ATP synthesis is generated via a membrane-bound electron transport chain originating with oxidation of the carbonyl group of acetate by $\mathrm{Cdh}$ and terminating with reduction of CoM-S-S-CoB by Hdr. Although the pathway of carbon flow from the methyl group of acetate to methane is understood for both aceticlastic genera, the understanding of electron transport coupled to generation of the proton gradient is incomplete. The majority of investigations have focused on Methanosarcina barkeri and Methanosarcina mazei for which electron transport is dependent on the production and consumption of $\mathrm{H}_{2}$ as an intermediate, although the great majority of Methanosarcina species [4] and all Methanosaeta species are unable to metabolize $\mathrm{H}_{2}$.

In the $\mathrm{H}_{2}$-metabolizing Methanosarcina species investigated, a ferredoxin accepts electrons from Cdh $[5,6]$ and donates to a membrane-bound Ech hydrogenase complex that produces $\mathrm{H}_{2}$ and generates a proton gradient for ATP synthesis [7-9]. A hypothesis has been advanced wherein $\mathrm{H}_{2}$ is re-oxidized by another membrane-bound hydrogenase (Vho) that transfers electrons to methanophenazine (MP), a quinone-like electron carrier [9]. In the model, MP donates electrons to the heterodisulfide reductase $\mathrm{HdrDE}$ accompanied by translocation of protons which further contributes to ATP synthesis.

An electron transport chain has been hypothesized for the marine isolate Methanosarcina acetivorans, the only non- $\mathrm{H}_{2}$-metabolizing acetotrophic methanogen for which the genome is sequenced. Although encoding $\mathrm{Cdh}$, the genome does not encode Ech hydrogenase $[10,11]$. Furthermore, in contrast to all $\mathrm{H}_{2}$-utilizing aceticlastic Methanosarcina species investigated [12], acetate-grown $M$. acetivorans synthesizes a six-subunit complex (Ma-Rnf) [13] encoded within a co-transcribed eight-gene (MA0658-0665) cluster with high identity to membrane-bound Rnf (hhodobacter $\underline{\text { nitrogen fixation) }}$ complexes from the domain Bacteria. It is hypothesized that the Ma-Rnf complex plays an essential role in the electron transport chain, generating a sodium gradient that is exchanged for a proton gradient driving ATP synthesis [13]. Consistent with this idea, it was recently shown that the six-subunit Rnf complex from Acetobacterium woodii of the domain Bacteria couples electron transport from reduced ferredoxin to $\mathrm{NAD}^{+}$with the generation of a sodium gradient [14]. Remarkably, the Ma-Rnf complex of $M$. acetivorans is co-transcribed with a gene (MA0658) encoding a multi-heme cytochrome $c$, and another flanking gene (MA0665) encoding a hypothetical membrane integral protein with unknown function [13]. Indeed, the cytochrome $c$ was shown to be synthesized in high levels of acetate-grown cells where it completely dominates the UV-visible spectrum of the purified membranes and is distinguishable from $b$-type cytochromes [13]. Furthermore, it was recently reported (A. M. Guss and W. W. Metcalf, unpublished results) that a six-subunit Ma-Rnf/cytochrome $c$ ( $\triangle M A 0658-0665)$ deletion mutant of $M$. acetivorans fails to grow with acetate [15]. However, biochemical evidence necessary to support the hypothesized role of cytochrome $c$ has not been forthcoming. The only other report of cytochromes $c$ in methanogens is for the $\mathrm{H}_{2}$-metabolizing species Methanosarcina mazei ( $f$. Methanosarcina strain Gö1) grown with methanol [16].

The freshwater isolate Methanosarcina thermophila is the only non- $\mathrm{H}_{2}$-metabolizing acetotrophic methanogen for which electron transport components have been investigated biochemically [17]. Like $\mathrm{H}_{2}$-metabolizing Methanosarcina species, ferredoxin mediates electron transfer between Cdh and the membrane-bound electron transport chain in which a cytochrome $b$ participates and dominates the UV-visible absorbance spectrum of membranes. It is also reported that MP is the electron donor to HdrDE [18]. Electron carriers other than cytochrome $b$ that participate between ferredoxin and MP were not identified. Importantly, no evidence for participation of Ma-Rnf or cytochrome $c$ was reported. Homologs encoding an Ma-Rnf complex and cytochrome $c$ are absent in the sequenced genome of Methanosaeta thermophila suggesting yet another novel electron transport chain that functions in the conversion of acetate to methane in this non- $\mathrm{H}_{2}$-metabolizing genus [19]. Clearly, diverse electron transport pathways have evolved in diverse acetotrophic methanogens necessitating biochemical investigations of representative species.

The absence of Ech hydrogenase and the demonstrated presence of the Ma-Rnf complex and cytochrome $c$ that is elevated in acetate-versus methanolgrown cells [13] suggests that electron transport of the non- $\mathrm{H}_{2}$-metabolizing marine isolate $M$. acetivorans is decidedly dissimilar from the genus Methanosaeta and $\mathrm{H}_{2}$-metabolizing acetotrophic species of the genus Methanosarcina. However, a biochemical investigation essential to support the role of electron carriers has not been reported for $M$. acetivorans. Here we report evidence indicating roles for ferredoxin, cytochrome $c$ and MP in electron transport of acetate-grown $M$. acetivorans. The results underscore the diversity of electron transport pathways in acetotrophic methanogens and contribute to a more complete understanding of acetotrophic methanogenesis. 


\section{Results}

The electron acceptor for the CO dehydrogenase/acetylCoA complex of $M$. acetivorans

The Cdh from acetate-grown $M$. acetivorans was purified to ascertain the electron acceptor that initiates electron transport. The $\mathrm{Cdh}$ complex purified from the $\mathrm{H}_{2}$ metabolizing acetotrophic species Methanosarcina barkeri contains five-subunits (CdhABCDE) [20] of which the CdhAE component oxidizes $\mathrm{CO}$ derived from the carbonyl group of acetate [21]. The genome of $M$. acetivorans is annotated with duplicate $\mathrm{Cdh}$ gene clusters [10], each encoding five subunits homologous to the Cdh subunits of $M$. barkeri. Previous proteomic analyses of acetate-grown $M$. acetivorans identified subunits CdhA, CdhB and CdhC from one cluster (MA1011-16) and CdhA, CdhB CdhC and CdhE from the other (MA3860-65) [22]. The purification was monitored by following the CO-dependent reduction of methyl viologen. SDS PAGE of the purified enzyme showed bands with molecular masses of $16 \mathrm{kDa}$ and $85 \mathrm{kDa}$ consistent with the predicted values for the CdhA and CdhE subunits encoded in the genome. Mass spectrometry of the protein bands identified the CdhA and CdhE subunits encoded by both $\mathrm{Cdh}$ gene clusters consistent with previous proteomic analyses that indicated up-regulation of both clusters in acetate-versus methanol-grown cells [22].

Ferredoxin from acetate-grown cells of $M$. acetivorans was purified as described in the Methods section to determine if it accepts electrons from the partially purified CdhAE components thereby initiating electron transport. Mass spectrometry analysis of the purified ferredoxin detected only one protein identified as the product of MA0431 previously annotated as a $2 \times$ [4Fe-4S] ferredoxin [23]. The UV-visible absorption spectrum of the as-purified protein was typical of ferredoxins with an absorption maximum at $402 \mathrm{~nm}$ that decreased upon reduction with dithionite (Additional file 1, Figure S1). The genome of $M$. acetivorans is annotated with nine genes encoding ferredoxins, a phylogenetic analysis of which is shown in Additional file 2, Figure S2. The analysis revealed that the product of MA0431 is closely related to the $2 \times[4 \mathrm{Fe}-4 \mathrm{~S}]$ ferredoxin purified from acetate-grown cells of $M$. thermophila [24-27] and the ferredoxin up-regulated in acetate-versus methanolgrown M. mazei [28]. These three ferredoxins contain two $\mathrm{CX}_{2} \mathrm{CX}_{2} \mathrm{CX}_{3} \mathrm{CP}$ motifs typical of $2 \times$ [4Fe-4S] ferredoxins and share high identity within a distinct clade (Additional file 2, Figure S2). Figure 1 shows CO-dependent reduction of the purified $M$. acetivorans ferredoxin catalyzed by the CdhAE components purified from $M$. acetivorans. These results suggest that ferredoxin isolated initiates the electron transport chain in both $M$.

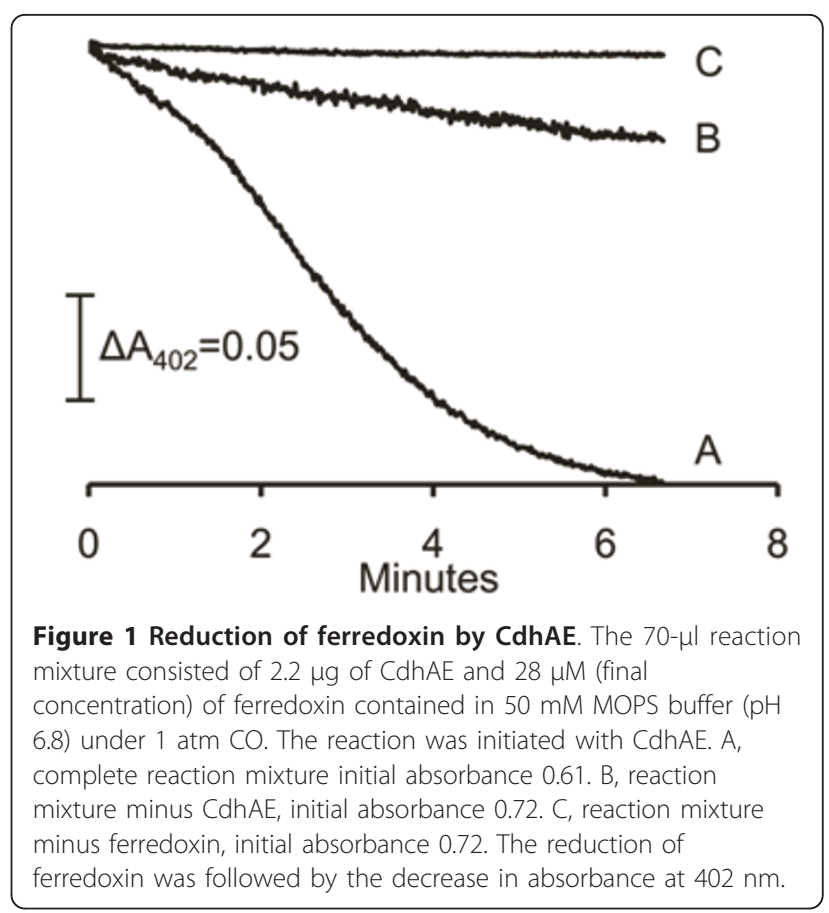

acetivorans and $\mathrm{H}_{2}$-metabolizing acetotrophic Methanosarcina species.

\section{Ferredoxin as the electron donor to the membrane- bound electron transport chain}

The finding that ferredoxin is an electron acceptor for the CdhAE component of the Cdh complex of $M$. acetivorans raises the question whether it is the direct electron donor to membrane-bound electron carriers or if other soluble electron carriers are required to mediate electron transfer between ferredoxin and the membrane. This question was addressed in a system containing sucrose gradient-purified membranes and plant ferredoxin-NADPH reductase (FNR) to regenerate reduced ferredoxin that was purified from acetate-grown cells. The CO-dependent reduction of ferredoxin with CdhAE was not used to avoid binding of $\mathrm{CO}$ to high spin heme in cytochrome $c$ and potentially inhibiting membranebound electron transport. The NADPH:CoM-S-S-CoB oxidoreductase activity was monitored by detecting the sulfhydryl groups of HS-CoM and HS-CoB (Figure 2). No significant activity was detected when each component of the reaction mixture was deleted individually including membranes. The dependence of the activity on purified membranes and the concentration of ferredoxin purified from acetate-grown $M$. acetivorans indicated a role for the ferredoxin in the direct transfer of electrons from CdhAE to the membrane-bound electron transport chain terminating with reduction of CoM-S-SCoB by heterodisulfide reductase. 


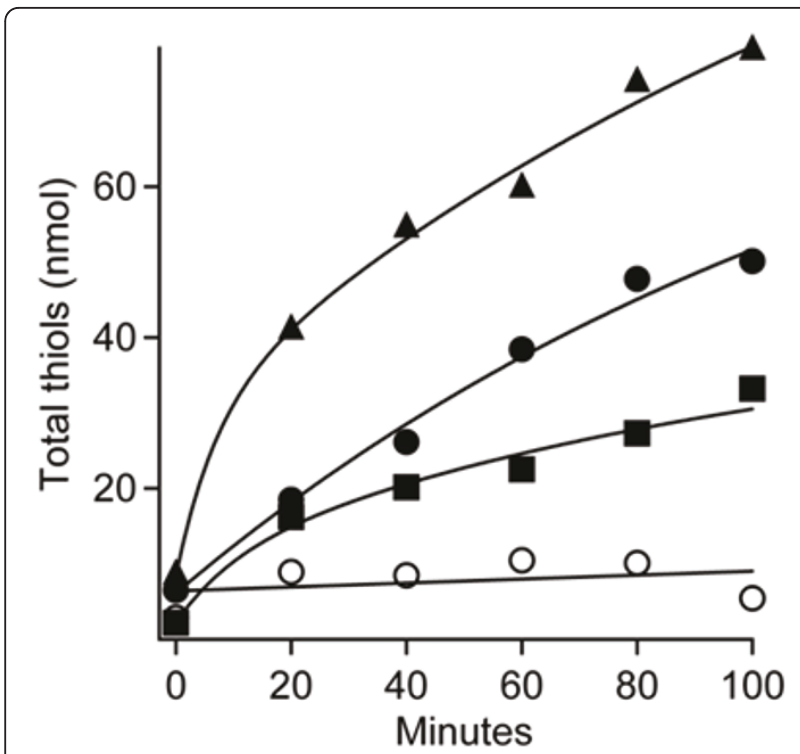

Figure 2 Ferredoxin:heterodisulfide oxidoreductase activity of membranes. The $100-\mu l$ reaction mixture consisted of $20 \mathrm{mM}$ $\mathrm{NADPH}, 2 \mu \mathrm{g}$ FNR (Sigma), the membrane fraction of acetate-grown cells ( $60 \mu \mathrm{g}$ protein), $1.1 \mathrm{mM}$ CoM-S-S-CoB and the indicated concentrations of ferredoxin contained in $50 \mathrm{mM}$ MOPS buffer $(\mathrm{pH}$ 6.8). Total thiols were determined by the DTNB assay. Symbols: (filled triangles) $1.2 \mu \mathrm{M}$ ferredoxin, (filled circles) $0.6 \mu \mathrm{M}$ ferredoxin, (filled squares) $0.3 \mu \mathrm{M}$ ferredoxin, (open circles) minus ferredoxin.

Role of cytochrome $c$ in the membrane-bound electron transport chain

It was previously documented [13] that purified membranes of acetate-grown $M$. acetivorans contain a multiheme cytochrome $c$ that clearly dominates the UV-visible spectrum of membranes from acetate-grown $M$. acetivorans with the major peak centered at $554 \mathrm{~nm}$ (Figure 3B). Absorbance at $554 \mathrm{~nm}$ increased on incubation of the membrane fraction with the reduced ferredoxin regenerating system indicating reduction of cytochrome $c$ that was dependent on ferredoxin (Figure 3). Addition of CoM-S-S$\mathrm{CoB}$ oxidized the reduced cytochrome (Figure 4) indicating that it is a component of the membrane-bound electron transport chain terminating with reduction of the heterodisulfide. The re-oxidation was too rapid to determine a rate and incomplete, albeit greater than $50 \%$. The explanation for incomplete re-oxidation is unknown, although the result is nearly identical to that reported for the re-oxidation of cytochromes in the membrane fraction of methanol-grown $M$. mazei that was rapid and reached $40 \%$ reoxidation [16]. This is the first report of cytochrome $c$ involvement in the conversion of acetate to methane.

\section{Role of methanophenazine in the membrane-bound} electron transport chain

The soluble analog of MP, 2-hydroxyphenazine, has been used to investigate the role of MP in methanogens

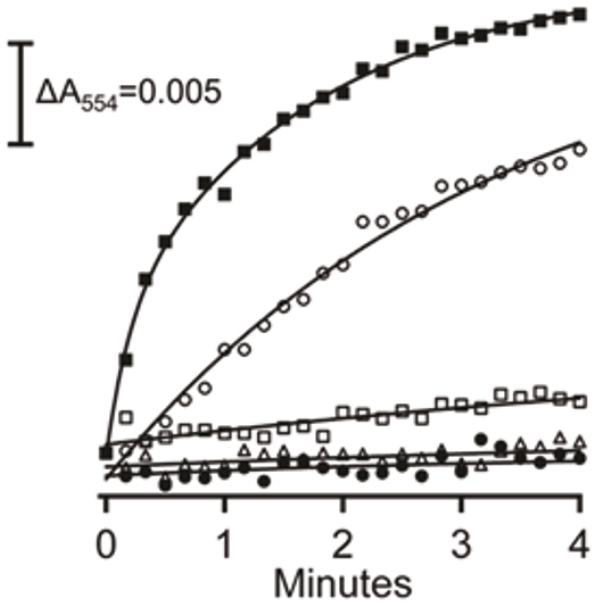

A

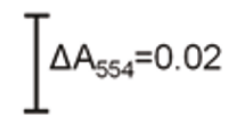

B

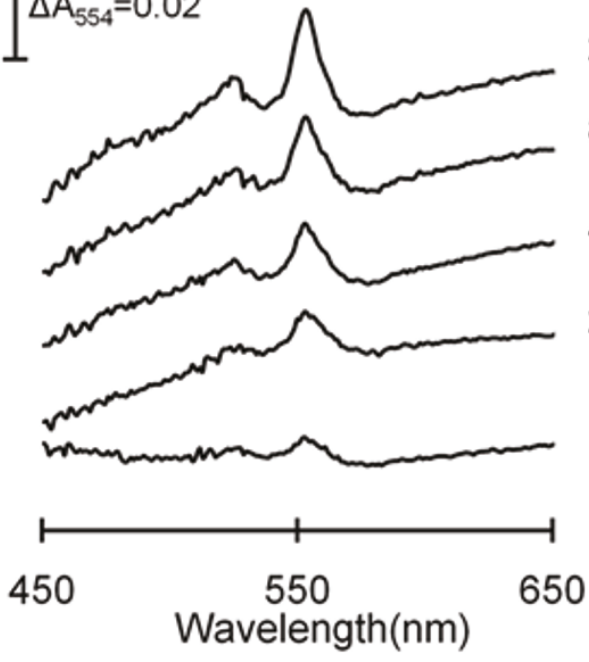

Figure 3 Ferredoxin-dependent reduction of membrane-bound cytochrome $\boldsymbol{c}$. The 100- $\mu$ l reaction mixture consisted of purified membranes (300 $\mu \mathrm{g}$ protein), the indicated amount of ferredoxin, 1 $\mu \mathrm{g} F N R$ and $1 \mathrm{mM}$ NADPH in $50 \mathrm{mM}$ MOPS (pH 6.8). The reaction was initiated by addition of FNR (Sigma). The reduction of cytochrome $c$ was followed at $554 \mathrm{~nm}$. Panel A, time-course for the reduction of cytochrome $c$. Symbols: (filled squares) $4 \mu \mathrm{M}$ ferredoxin; (open circles) $0.2 \mu \mathrm{M}$ ferredoxin; (open squares) minus ferredoxin; (open triangles) minus FNR; (filled circles) minus NADPH. Panel $B$, reduced minus oxidized spectra recorded at the indicated times after initiation of the reaction containing $4 \mu \mathrm{M}$ ferredoxin.

$[18,29]$. The 2-hydroxyphenazine that was reduced in the presence of membranes with the $\mathrm{CO} / \mathrm{CdhAE}$ ferredoxin regenerating system was re-oxidized upon addition of CoM-S-S-CoB (Figure 5) consistent with a membrane-bound heterodisulfide reductase. The re-oxidation generated a total of $239 \mu \mathrm{M}$ free thiol groups in this representative experiment, a result that is in approximate agreement with the observed oxidation of $106 \mu \mathrm{M}$ 2-hydroxyphenazine. Assuming a two-electron 


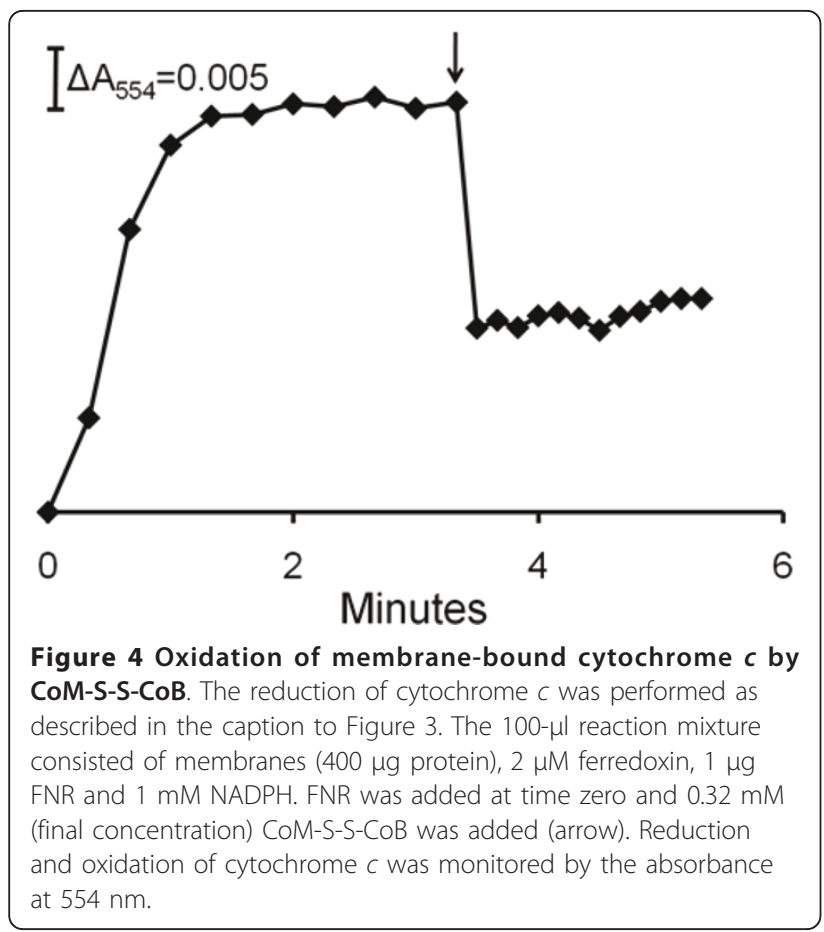

transfer from the MP analog, $212 \mu \mathrm{M}$ free thiol groups would be expected. These results indicate that MP is a component of the membrane-bound electron transport chain terminating with reduction of CoM-S-S-CoB.

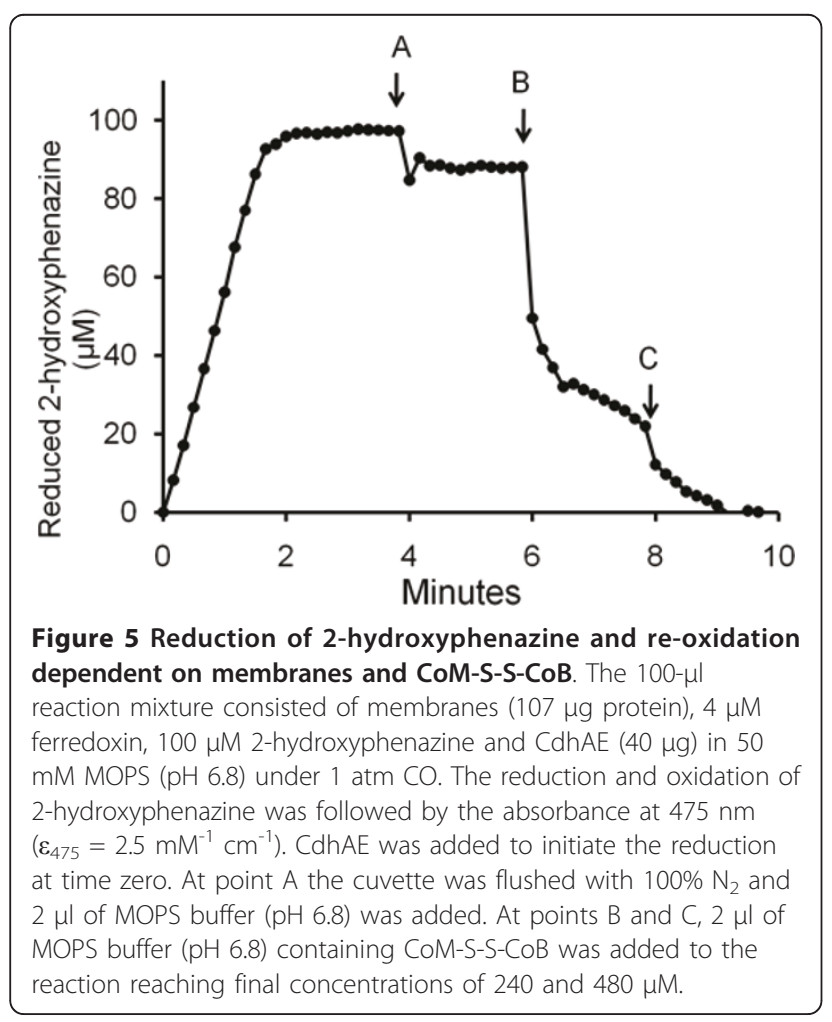

The results implicating MP and cytochrome $c$ in the membrane-bound electron transport chain presents the possibility of electron transfer between these carriers. The MP analog 2-hydroxyphenazine re-oxidized cytochrome $c$ when added to membranes of acetate-grown cells previously reduced with ferredoxin (Figure 6). These results suggest that MP is either directly or indirectly linked to cytochrome $c$, a result further supporting the participation of MP and cytochrome $c$ in the membrane-bound electron transport chain.

\section{Discussion}

The overwhelming majority of methanogens capable of growth via conversion of the methyl group of acetate to

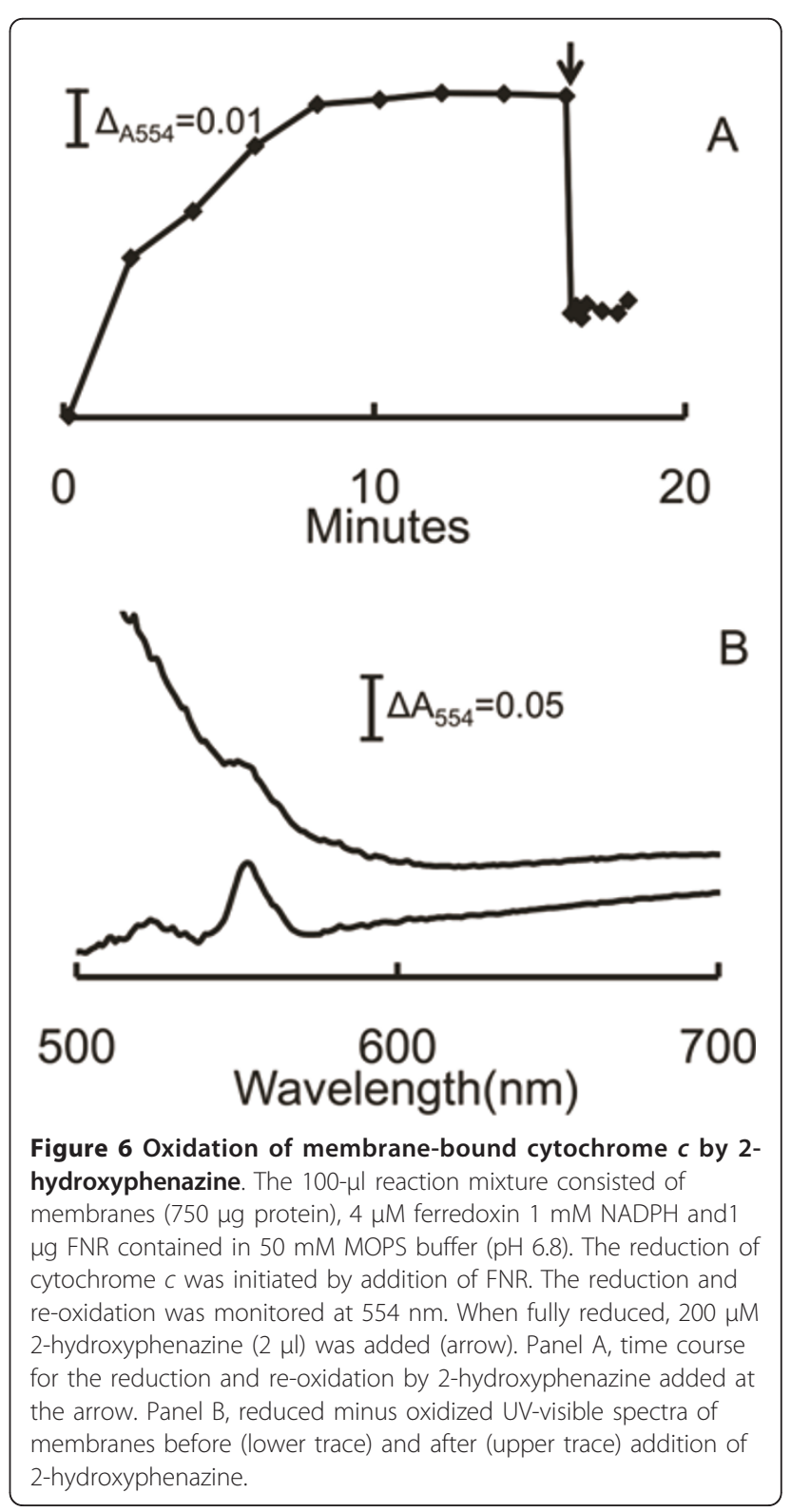


methane do not metabolize $\mathrm{H}_{2}$ suggesting they employ an electron transport pathway distinct from that proposed for the few acetotrophic methanogens in which $\mathrm{H}_{2}$ is an obligatory intermediate. M. acetivorans is the ideal candidate to represent the majority of acetotrophic Methanosarcina species by virtue of its sequenced genome and published proteomic analyses leading to the hypothesis of a novel electron transport pathway for acetotrophic methanogens incapable of metabolizing $\mathrm{H}_{2}$. This, the first biochemical investigation of electron transport in $M$. acetivorans, has established roles for electron carriers that reveal both commonalities and differences in electron transport pathways of diverse acetotrophic Methanosarcina species.

Figure 7 compares the current understanding of electron transport for acetate-grown $M$. acetivorans with that for $\mathrm{H}_{2}$-metabolizing acetotrophic Methanosarcina species. In both pathways, the five-subunit CdhABCDE complex (not shown) cleaves the $\mathrm{C}-\mathrm{C}$ and $\mathrm{C}-\mathrm{S}$ bonds of acetyl-CoA releasing a methyl group and $\mathrm{CO}$ that is oxidized to $\mathrm{CO}_{2}$ with electrons transferred to ferredoxin. The CdhAE component of $M$. acetivorans was isolated independently from the other subunits and both copies encoded in the genome were represented. Although it was not possible to determine which CdhAE component reduced ferredoxin, the high percent identities $(\mathrm{CdhA}$, MA1016 vs. MA3860 = 84\% and CdhE, MA1015 vs. MA3861 $=82 \%$ ) suggests it is the electron acceptor for either or both copies. In both pathways, ferredoxin is the electron donor to a membrane-bound electron transport chain that terminates with MP donating electrons to the heterodisulfide reductase $\mathrm{HdrDE}$ that catalyzes the reduction of CoB-S-S-CoM. Proteomic and genetic evidence $[15,22]$ indicates that $\mathrm{HdrDE}$ functions in acetate-grown $M$. acetivorans. MP is the direct electron donor to $\mathrm{HdrDE}$ in acetate-grown cells of $\mathrm{H}_{2}$-metabolizing Methanosarcina species and the non- $\mathrm{H}_{2}$ metabolizing $M$. thermophila [18]. Thus, it is reasonable to postulate that MP is also the direct electron donor to HdrDE of $M$. acetivorans. However, the electron transport pathways of $\mathrm{H}_{2}$-metabolizing and non- $\mathrm{H}_{2}$-metabolizing species diverge significantly in electron transfer between ferredoxin and MP. In $\mathrm{H}_{2}$-metabolizing species, ferredoxin donates electrons to the membrane-bound Ech hydrogenase. A $\mathrm{H}_{2}$ cycling mechanism is postulated in which the $\mathrm{H}_{2}$ generated by Ech hydrogenase is re-oxidized by the MP-reducing Vho-type hydrogenase further contributing to the proton gradient [8]. Although the genome of $M$. acetivorans contains homologs of genes encoding Vho-type hydrogenases they are not expressed during growth with acetate [4], a result consistent with the absence of Ech hydrogenase and inability to metabolize $\mathrm{H}_{2}$. Instead, the results reported here support a role for cytochrome $c$ mediating electron transport between ferredoxin and MP, although the identities of the direct electron donor and acceptor for cytochrome $c$ remain

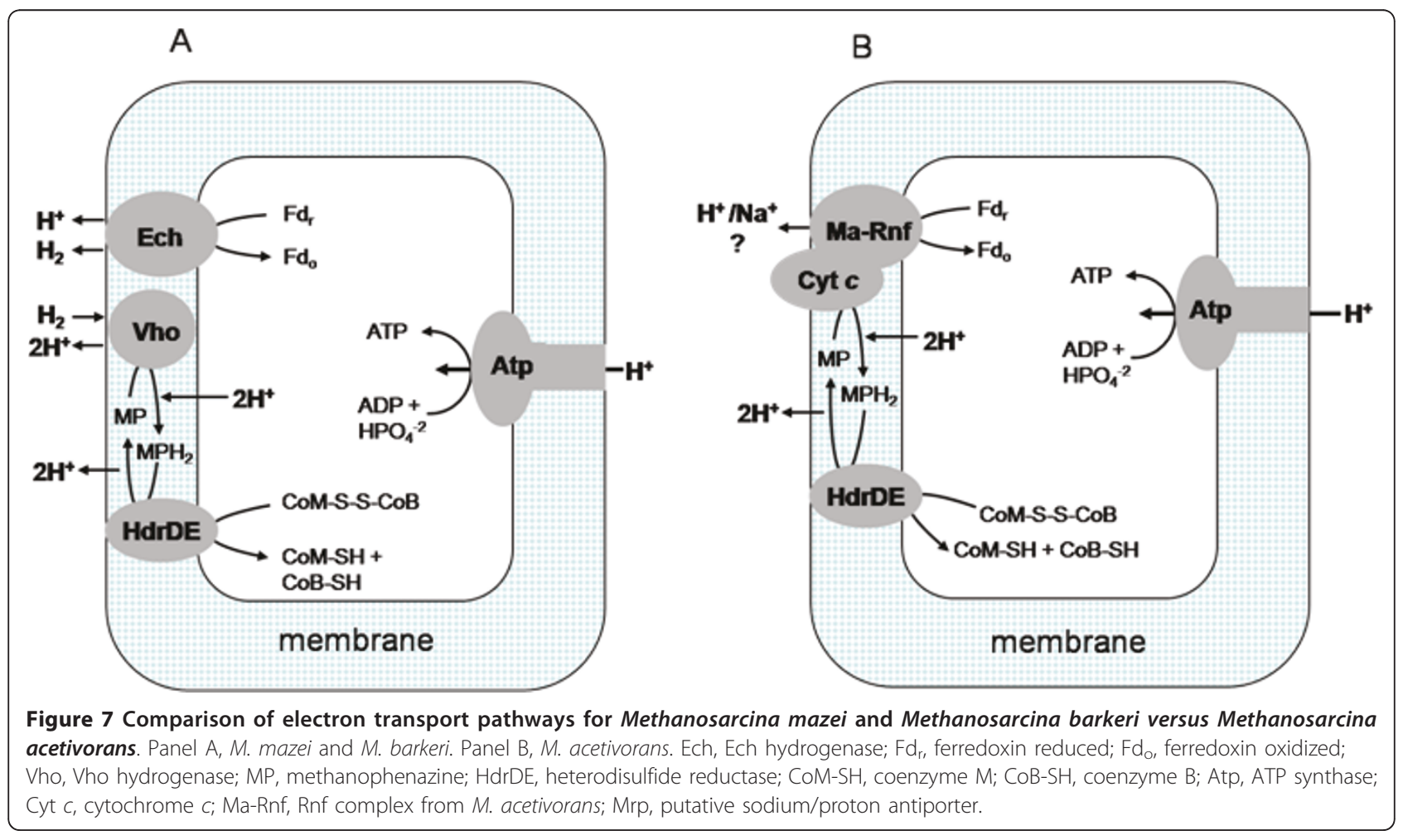


unknown. The membrane location of cytochrome $c$ is unknown; however, if on the outer aspect as for multiheme cytochromes $c$ in the domain Bacteria, ferredoxin would be an unlikely electron donor. The most probable electron donor to cytochrome $c$ is the Ma-Rnf complex that is also hypothesized to accept electrons from ferredoxin in analogy to homologous Rnf complexes from the domain Bacteria [13,30]. In the absence of biochemical evidence, the proposed role for Ma-Rnf in electron transport is at least consistent with up-regulation in acetate $v s$. methanol-grown cells and the reported failure of an Ma-Rnf-cytochrome $c$ deletion mutant ( $\triangle M A 0658$ 0665) of $M$. acetivorans to grow with acetate [15]. The proposed interaction of Ma-Rnf with cytochrome $c$ is supported by co-transcription of the encoding genes and up-regulation in acetate- $v s$. methanol-grown cells [13]. A role for cytochrome $c$ in the electron transport chain is also supported by results showing re-oxidation of cytochrome $c$ upon addition of the MP analog 2-hydroxyphenazine to ferredoxin-reduced membranes, although an unknown carrier mediating electron transfer between cytochrome $c$ and MP cannot be ruled out.

It was recently shown that the $\mathrm{Rnf}$ complex from $A$. woodii translocates sodium ions coupled to electron transfer from ferredoxin to $\mathrm{NAD}^{+}$[14]. In view of the potential sodium ion pumping function of Ma-Rnf, it is interesting to note that a multi-subunit sodium/proton antiporter (Mrp) is up-regulated in acetate-grown $M$. acetivorans and that the encoding genes are absent in $\mathrm{H}_{2}$-metabolizing Methanosarcina species [13]. Thus, it is tempting to speculate that Ma-Rnf generates a sodium gradient (high outside) that is exchanged for a proton gradient by Mrp. The only other coupling site is the reduction and oxidation of MP generating a proton gradient as proposed for $\mathrm{H}_{2}$-metabolizing Methanosarcina species (Figure 7). The role of a proton gradient driving ATP synthesis is consistent with the presence of a proton translocating ATP synthase in acetate-grown cells [13] recently shown to be the primary ATP synthase [31].

The available evidence indicates that the non- $\mathrm{H}_{2}$ metabolizing freshwater isolate $M$. thermophila also utilizes ferredoxin as electron donor to a membrane-bound electron transport chain involving cytochrome $b$ and culminating with MP donating electrons to $\mathrm{HdrDE}$ $[17,18,32]$; however, a role for cytochrome $c$ is not evident and other electron carriers have not been reported. Thus, based on current evidence, it appears that all acetotrophic Methanosarcina species have in common ferredoxin as electron donor to a membrane-bound electron transport chain terminating with MP donating electrons to HdrDE, although differ widely in membrane components transferring electrons from ferredoxin to MP. The evidence for involvement of $\mathrm{HdrDE}$ in acetate-grown cells is convincing; however, genes (MA2868, MA4236 and MA4237) homologous to those encoding the soluble $\mathrm{HdrABC}$ heterodisulfide reductase of $\mathrm{CO}_{2}$-reducing methanogens were shown to be up regulated in acetateversus methanol-grown $M$. acetivorans [33]. This result is consistent with the previously reported increased abundance of HdrA encoded by MA2868 in acetateversus methanol-grown $M$. acetivorans [22] which opens the possibility that the electron transport chain may terminate with both the membrane HdrDE or a soluble $\mathrm{Hdr} A B C$ heterodisulfide reductase.

Of the nine putative $2 \times$ [4Fe-4S] ferredoxins annotated for the genome of $M$. acetivorans, only the ferredoxin encoded by MA0431 was purified from acetategrown cells. While it cannot be ruled out that other ferredoxins are synthesized in acetate-grown cells, the results suggest that the ferredoxin encoded by MA0431 is at least dominant in acetate-grown cells. Of the nine putative $2 \times[4 \mathrm{Fe}-4 \mathrm{~S}]$ ferredoxins, the one purified from $M$. acetivorans is most closely related to that isolated from acetate-grown $M$. thermophila [26], a result suggesting it is the preferred electron acceptor of CdhAE in acetate-grown Methanosarcina species.

Interestingly, genes encoding subunits of Ma-Rnf or Ech hydrogenase are absent in the genome of the acetate-utilizing isolate Methanosaeta thermophila [19] that is also incapable of metabolizing $\mathrm{H}_{2}$ suggesting still other alternative electron transport pathways coupled to generation of ion gradients driving ATP synthesis in acetate-utilizing methanogens. The physiological significance of these diverse electron transport pathways is yet to be determined; however, it has been suggested that avoiding $\mathrm{H}_{2}$ is advantageous to the marine isolate $M$. acetivorans since sulfate reducing species that dominate this environment outcompete methanogens for $\mathrm{H}_{2}$ potentially disrupting electron transport [13]. It is important to note here that although $M$. acetivorans is incapable of growth with $\mathrm{H}_{2} / \mathrm{CO}_{2}$ it synthesizes all of the enzymes necessary for reduction of $\mathrm{CO}_{2}$ to methane and is capable of robust growth via the $\mathrm{CO}_{2}$-reduction pathway albeit with electrons derived from the oxidation of $\mathrm{CO}$ [34-36].

\section{Comparative analysis of the $M$. thermophila genome}

$M$. thermophila is an acetotrophic Methanosarcina species incapable of metabolizing $\mathrm{H}_{2}[37,38]$. Analysis of the genomic sequence revealed a gene cluster identical in arrangement and homologous to genes encoding the six subunits of Ma-Rnf and multi-heme cytochrome $c$ of $M$. acetivorans with deduced sequence identities ranging from 86 to 98\% (Additional file 3, Figure S3A). Alignments of the deduced sequences showed strict conservation of heme-binding, flavin binding and iron-sulfur binding motifs suggesting conserved functions 
(Additional file 3, Figure S3B). Although not conclusive, these results are consistent with a role for the Ma-Rnf complex and multi-heme cytochrome $c$ in the electron transport pathway of $M$. thermophila grown with acetate. Furthermore, the genome of $M$. thermophila contains a gene cluster (Additional file 4, Figure S4) homologous to genes encoding the seven subunits of the sodium/proton antiporter (Mrp) that is up-regulated in acetate-versus methanol-grown cells of $M$. acetivorans and absent in the sequenced genomes of acetotrophic Methanosarcina species capable of metabolizing $\mathrm{H}_{2} / \mathrm{CO}_{2}$ [22,39].

\section{Conclusions}

Although the majority of Methanosarcina species are unable to metabolize $\mathrm{H}_{2}$, electron transport has only been investigated in the few species for which $\mathrm{H}_{2}$ is an obligatory intermediate. $M$. acetivorans is proposed to utilize a fundamentally different electron transport pathway based on bio-informatic, proteomic and genetic approaches. However, the proposal has not been tested biochemically. The results indicate roles for ferredoxin, cytochrome $c$ and MP in support of the proposed electron transport pathway. Further, this is the first report for involvement of a cytochrome $c$ in acetotrophic methanogens. The results suggest that diverse acetotrophic Methanosarcina species have evolved diverse membrane-bound electron transport pathways leading from ferredoxin and culminating with MP donating electrons to HdrDE for reduction of CoM-S-S-CoB.

\section{Methods \\ Materials}

CoM-S-S-CoB was a kind gift of Dr. Jan Keltjens. 2hydroxyphenazine was custom synthesized by SigmaAldrich (St. Louis, MO). All other chemicals were purchased from Sigma-Aldrich or VWR International (West Chester, PA). All chromatography columns, resins and pre-packed columns were purchased from GE Healthcare (Waukesha, WI).

\section{Preparation of cell extract and membranes}

M. acetivorans [40] was cultured with acetate as described previously [41] and the cell paste was frozen at $-80^{\circ} \mathrm{C}$. All solutions were $\mathrm{O}_{2}$-free and manipulations were performed anaerobically in an anaerobic chamber (Coy Manufacturing, Ann Arbor, MI) containing 95\% $\mathrm{N}_{2}$ and $5 \% \mathrm{H}_{2}$. Frozen cells were thawed, re-suspended (1 g wet weight $/ \mathrm{ml}$ buffer) in $50 \mathrm{mM}$ MOPS buffer $(\mathrm{pH}$ $6.8)$ containing $10 \%(\mathrm{v} / \mathrm{v})$ ethylene glycol and passed twice through a French pressure cell at $6.9 \times 10^{3} \mathrm{kPa}$. The lysate was centrifuged at 7,200 $\times g$ for $15 \mathrm{~min}$ to pellet cell debris and unbroken cells. Membranes were purified from the cell extract using a discontinuous sucrose gradient comprised of $2 \mathrm{ml} 70 \%$ sucrose, $4 \mathrm{ml}$ $30 \%$ sucrose and $1.5 \mathrm{ml} 20 \%$ sucrose contained in 50 mM MOPS buffer ( $\mathrm{pH}$ 6.8). A $2 \mathrm{ml}$ volume of cell extract was overlaid on the gradient and centrifuged at $200,000 \times g$ for $2 \mathrm{~h}$ in a Beckman type $50 \mathrm{Ti}$ rotor. The brown band containing membranes at the $30 \%$ and $70 \%$ sucrose interface was collected and stored at $-80^{\circ} \mathrm{C}$ until use.

\section{Purification of the $\alpha \varepsilon$ component (CdhAE) of the CO dehydrogenase/acetyl-CoA synthase complex}

All purification steps and biochemical assays were performed anaerobically in the anaerobic chamber. Crude cell extract of acetate-grown $M$. acetivorans was centrifuged at $200,000 \times g$ for $2 \mathrm{~h}$ to pellet the membrane fraction. The supernatant solution (200 $\mathrm{mg}$ of protein in $10-\mathrm{ml}$ ) containing the soluble fraction was loaded onto a Q-Sepharose FF column (50 ml bed volume) equilibrated with $50 \mathrm{mM}$ MOPS ( $\mathrm{pH}$ 6.8). The column was developed with $500 \mathrm{ml}$ of a $0-1.0 \mathrm{M} \mathrm{NaCl}$ linear gradient. Each $10 \mathrm{ml}$ fraction was assayed for $\mathrm{CO}$ dehydrogenase activity by monitoring the $\mathrm{CO}$-dependent reduction of methyl viologen as previously described [42]. The pooled fractions from the peak with the highest specific activity were concentrated 10-fold with a Vivacell 70 protein concentrator equipped with a 10kDa cut off membrane (Sartorius Group, Göttingen, Germany). A $1.0 \mathrm{M}$ solution of $\left(\mathrm{NH}_{4}\right)_{2} \mathrm{SO}_{4}$ contained in $50 \mathrm{mM}$ MOPS ( $\mathrm{pH}$ 6.8) was added to the concentrated protein solution to final concentration of $900 \mathrm{mM}$ and loaded onto a Phenyl-Sepharose FF (low sub) column (20-ml bed volume) equilibrated with $50 \mathrm{mM}$ MOPS ( $\mathrm{pH}$ 6.8) containing $1.0 \mathrm{M}\left(\mathrm{NH}_{4}\right)_{2} \mathrm{SO}_{4}$. The column was developed with $100 \mathrm{ml}$ of a 1.0-0.0 $\mathrm{M}\left(\mathrm{NH}_{4}\right)_{2} \mathrm{SO}_{4}$ decreasing linear gradient. Fractions from the peak of $\mathrm{CO}$ dehydrogenase activity were pooled and concentrated followed by addition of a volume of $50 \mathrm{mM}$ MOPS ( $\mathrm{pH}$ 6.8) to lower the $\left(\mathrm{NH}_{4}\right)_{2} \mathrm{SO}_{4}$ concentration to below $100 \mathrm{mM}$ and then loaded on a HiTrap QSepharose HP column ( $5 \mathrm{ml}$ bed volume) equilibrated with $50 \mathrm{mM}$ MOPS buffer ( $\mathrm{pH}$ 6.8). The column was developed with $50 \mathrm{ml}$ of a $0-1.0 \mathrm{M} \mathrm{NaCl}$ linear gradient. The peak containing $\mathrm{CO}$ dehydrogenase activity that eluted at approximately $0.3 \mathrm{M} \mathrm{NaCl}$ was collected and stored at $-80^{\circ} \mathrm{C}$ until use.

\section{Purification of ferredoxin}

All purification steps and biochemical assays were performed anaerobically in the anaerobic chamber. Ferredoxin was assayed by the ability to couple $\mathrm{CO}$ oxidation by $\mathrm{CdhAE}$ to the reduction of metronidazole followed by the decrease in $A_{320}\left(\varepsilon_{320}=9300 \mathrm{M}^{-1} \mathrm{~cm}^{-1}\right)$ similar to that described previously [27]. One unit of activity was the amount that reduced $1 \mu \mathrm{mol}$ of metronidazole/ 
min. The reaction mixture $(100 \mu \mathrm{l})$ contained $100 \mu \mathrm{M}$ metronidazole and 1-3 $\mu \mathrm{g}$ CdhAE in $50 \mathrm{mM}$ Tris buffer ( $\mathrm{pH}$ 8.0) to which $1-10 \mu \mathrm{l}$ of the column fraction was added. The reaction was contained in an anaerobic cuvette flushed with $100 \%$ CO.

The soluble fraction of cell extract from acetate-grown $M$. acetivorans was loaded onto a Q-sepharose FF column (20 ml bed volume) equilibrated with $50 \mathrm{mM}$ MOPS (pH 6.8) containing $10 \%(\mathrm{v} / \mathrm{v})$ ethylene glycol. The column was developed with $200 \mathrm{ml}$ of a $0-1.0 \mathrm{M}$ linear $\mathrm{NaCl}$ gradient. The fraction with the highest activity was then diluted 10fold with $50 \mathrm{mM}$ MOPS (pH 6.8) containing 10\% (v/v) ethylene glycol. The solution was loaded on a Mono Q column (1.7 ml bed volume) to which $10 \mathrm{ml}$ of a $0-1.0 \mathrm{M}$ $\mathrm{NaCl}$ linear gradient was applied. The fraction containing ferredoxin that eluted at $600 \mathrm{mM} \mathrm{NaCl}$ was loaded on a Sephadex G-75 gel filtration column (100 ml bed volume) and developed with $50 \mathrm{mM}$ MOPS (pH 6.8) containing $10 \%(\mathrm{v} / \mathrm{v})$ ethylene glycol and $150 \mathrm{mM} \mathrm{NaCl}$. The peak containing the purified ferredoxin was concentrated to $\mathrm{A}_{402}>0.2$ with a Vivacell 70 protein concentrator equipped with a $5-\mathrm{kDa}$ cutoff membrane and stored at $-80^{\circ} \mathrm{C}$ until use. The protein concentration was estimated by the ratio of absorbance at 230 and $260 \mathrm{~nm}$ as described [43].

\section{Analytical}

All protein concentrations except for ferredoxin were determined by the bicinchoninic acid assay using the reagent from Thermo Scientific, Inc.. Detection of the free sulfhydryl groups of CoM-SH and CoB-SH was performed as previously described [17]. The buffer used in the assay was $25 \mathrm{mM}$ sodium acetate containing $1 \mathrm{mM}$ DTNB (5,5'-dithiobis-(2-nitrobenzoic acid)). All assays in this study were performed anaerobically with vacuum degassed solutions contained in sealed cuvettes with the indicated atmosphere and at room temperature.

\section{Nucleotide sequence accession number}

The sequences of DNA encoding Rnf and Mrp of $M$. thermophila have been deposited in the GenBank database under accession number JN173061, JN173062, JN173063, JN173064, JN173065, JN173066, JN173067, JN173068, JN173069, JN173070, JN173071, JN173072, JN173073, JN173074, JN173075.

\section{Additional material}

Additional file 1: Figure S1. UV-visible absorption spectra of purified ferredoxin. As-purified (-), dithionite reduced (...). The protein concentration was $20 \mu \mathrm{M}$.

Additional file 2: Figure S2. Phylogenetic analysis and sequence alignment of ferredoxins. The $M$. mazei and M. acetivorans sequences, labeled with the prefix MA, were derived from the CMR database [23]. The M. thermophila (M.t.) sequence is published [26]. The sequence of the $2 \times[4 \mathrm{Fe}-4 \mathrm{~S}$ ] Clostridium pasteurianum is published [44] and the sequence of the $2 \mathrm{Fe}-2 \mathrm{~S}$ Spinacia oleracea ferredoxin was obtained from the NCBI database (accession number O04683). Panel A, Phylogenetic analysis of ferredoxins. The tree was constructed by the neighbor-joining method with the MEGA4 program [45]. Bootstrap values are shown at the nodes. Bar, evolutionary distance of 0.2. Panel B, Sequence alignment of ferredoxins from Methanosarcina species. Motifs predicted to ligate two $4 \mathrm{Fe}-4 \mathrm{~S}$ clusters are highlighted. The alignment was performed with ClustalX2 [46].

Additional file 3: Figure S3. Comparison of rnf genes between Methanosarcina thermophila and Methanosarcina acetivorans. Panel A. Organization of rnf genes in Methanosarcina thermophila versus Methanosarcina acetivorans. Numbers next to the arrows indicate deduced sequence identity. Panel B. Alignment of the deduced sequences of rnf genes between Methanosarcina thermophila (Mt) and Methanosarcina acetivorans (Ma). Highlighted are: conserved heme binding sites ( $\mathrm{CXXCH}$ and $\mathrm{CXXXCH}$ ) in $\mathrm{Cyt} C$, the flavin binding motif (SGAT) in RnfG, and cysteine motifs binding iron-sulfur clusters in RnfC and RnfB.

Additional file 4: Figure S4. Alignment of $m r p$ gene clusters between Methanosarcina thermophila and Methanosarcina acetivorans. Numbers next to the arrows indicate deduced sequence identity.

\section{Acknowledgements}

This work was supported by the National Science Foundation. We thank Dr. Jan Keltjens for generously supplying CoM-S-S-CoB and the Penn StateHershey Core Research Facilities for mass spectrometry analyses.

\section{Author details}

'Department of Biochemistry and Molecular Biology, Eberly College of Science, The Pennsylvania State University, University Park, Pennsylvania 16802-4500, USA. E. I. DuPont de Nemours Company, Central Research and Development, Experimental Station, Wilmington, Delaware 19880, USA.

\section{Authors' contributions}

MW carried out the biochemical studies, participated in sequence analysis and drafted the manuscript. J-F T carried out the genomic sequencing and sequence alignments. JGF conceived of the study, participated in its design and coordination, and finalized the manuscript. All authors read and approved the final manuscript.

\section{Competing interests}

The authors declare that they have no competing interests.

Received: 13 April 2011 Accepted: 24 July 2011 Published: 24 July 2011

\section{References}

1. Liu Y, Whitman WB: Metabolic, phylogenetic, and ecological diversity of the methanogenic archaea. Ann N Y Acad Sci 2008, 1125:171-189.

2. Ferry JG: How to make a living exhaling methane. Annu Rev Microbiol 2010, 64:453-473.

3. Thauer RK, Kaster AK, Seedorf H, Buckel W, Hedderich R: Methanogenic archaea: ecologically relevant differences in energy conservation. Nat Rev Microbiol 2008, 6:579-591.

4. Guss AM, Kulkarni G, Metcalf WW: Differences in hydrogenase gene expression between Methanosarcina acetivorans and Methanosarcina barkeri. Journal of Bacteriology 2009, 191(8):2826-2833.

5. Meuer J, Kuettner HC, Zhang JK, Hedderich R, Metcalf WW: Genetic analysis of the archaeon Methanosarcina barkeri Fusaro reveals a central role for Ech hydrogenase and ferredoxin in methanogenesis and carbon fixation. Proc Natl Acad Sci USA 2002, 99(8):5632-5637.

6. Fischer R, Thauer RK: Ferredoxin-dependent methane formation from acetate in cell extracts of Methanosarcina barkeri (strain MS). FEBS Lett 1990, 269:368-372.

7. Meuer J, Bartoschek S, Koch J, Kunkel A, Hedderich R: Purification and catalytic properties of Ech hydrogenase from Methanosarcina barkeri. Eur J Biochem 1999, 265(1):325-335. 
8. Welte C, Kratzer C, Deppenmeier U: Involvement of Ech hydrogenase in energy conservation of Methanosarcina mazei. FEBS J 2010, 277(16):3396-3403.

9. Welte C, Kallnik V, Grapp M, Bender G, Ragsdale S, Deppenmeier U: Function of Ech hydrogenase in ferredoxin-dependent, membranebound electron transport in Methanosarcina mazei. Journal of Bacteriology 2010, 192(3):674-678

10. Galagan JE, Nusbaum C, Roy A, Endrizzi MG, Macdonald P, FitzHugh W, Calvo $S$, Engels $R$, Smirnov $S$, Atnoor $D$, et al: The genome of $M$. acetivorans reveals extensive metabolic and physiological diversity. Genome Res 2002, 12(4):532-542.

11. Nelson MJK, Ferry JG: Carbon monoxide-dependent methyl coenzyme M methylreductase in acetotrophic Methanosarcina spp. Journal of Bacteriology 1984, 160:526-532.

12. Deppenmeier U, Muller V: Life close to the thermodynamic limit: how methanogenic archaea conserve energy. Results Probl Cell Differ 2008, 45:123-152.

13. Li Q, Li L, Rejtar T, Lessner DJ, Karger BL, Ferry JG: Electron transport in the pathway of acetate conversion to methane in the marine archaeon Methanosarcina acetivorans. J Bacteriol 2006, 188(2):702-710.

14. Biegel E, Müller V: Bacterial Na+-translocating ferredoxin:NAD+ oxidoreductase. Proc Natl Acad Sci USA 2010, 107:18138-18142.

15. Buan NR, Metcalf WW: Methanogenesis by Methanosarcina acetivorans involves two structurally and functionally distinct classes of heterodisulfide reductase. Mol Microbiol 2010, 75:843-853.

16. Kamlage B, Blaut M: Characterization of cytochromes from Methanosarcina strain Go1 and their involvement in electron transport during growth on methanol. Journal of Bacteriology 1992, 174:3921-3927.

17. Peer CW, Painter MH, Rasche ME, Ferry JG: Characterization of a CO: heterodisulfide oxidoreductase system from acetate-grown Methanosarcina thermophila. Journal of Bacteriology 1994, 176:6974-6979.

18. Murakami E, Deppenmeier U, Ragsdale SW: Characterization of the intramolecular electron transfer pathway from 2-hydroxyphenazine to the heterodisulfide reductase from Methanosarcina thermophila. J Biol Chem 2001, 276:2432-2439.

19. Smith KS, Ingram-Smith C: Methanosaeta, the forgotten methanogen? Trends Microbiol 2007, 7:150-155.

20. Grahame DA: Catalysis of acetyl-CoA cleavage and tetrahydrosarcinapterin methylation by a carbon monoxide dehydrogenase-corrinoid enzyme complex. J Biol Chem 1991, 266:22227-22233.

21. Gong W, Hao B, Wei Z, Ferguson DJ Jr, Tallant T, Krzycki JA, Chan MK: Structure of the a2e2 Ni-dependent CO dehydrogenase component of the Methanosarcina barkeri acetyl-CoA decarbonylase/synthase complex. Proc Natl Acad Sci USA 2008, 105(28):9558-9563.

22. Li L, Li Q, Rohlin L, Kim U, Salmon K, Rejtar T, Gunsalus RP, Karger BL, Ferry JG: Quantitative proteomic and microarray analysis of the archaeon Methanosarcina acetivorans grown with acetate versus methanol. J Proteome Res 2007, 6(2):759-771.

23. The Comprehensive Microbial Resource. J Craig Venter Institute 2011 [http://cmr.tigr.org/tigr-scripts/CMR/CmrHomePage.cgi].

24. Clements AP, Kilpatrick L, Lu WP, Ragsdale SW, Ferry JG: Characterization of the iron-sulfur clusters in ferredoxin from acetate-grown Methanosarcina thermophila. Journal of Bacteriology 1994, 176:2689-2693.

25. Terlesky KC, Ferry JG: Purification and characterization of a ferredoxin from acetate-grown Methanosarcina thermophila. J Biol Chem 1988, 263:4080-4082.

26. Clements AP, Ferry JG: Cloning, nucleotide sequence, and transcriptional analyses of the gene encoding a ferredoxin from Methanosarcina thermophila. Journal of Bacteriology 1992, 174:5244-5250.

27. Terlesky $\mathrm{KC}$, Ferry JG: Ferredoxin requirement for electron transport from the carbon monoxide dehydrogenase complex to a membrane-bound hydrogenase in acetate-grown Methanosarcina thermophila. J Biol Chem 1988, 263:4075-4079.

28. Hovey R, Lentes S, Ehrenreich A, Salmon K, Saba K, Gottschalk G, Gunsalus RP, Deppenmeier U: DNA microarray analysis of Methanosarcina mazei Go1 reveals adaptation to different methanogenic substrates. Mol Genet Genomics 2005, 273:225-239.

29. Abken HJ, Tietze M, Brodersen J, Baumer S, Beifuss U, Deppenmeier U: Isolation and characterization of methanophenazine and the function of phenazines in membrane-bound electron transport of Methanosarcina mazei Go1. Journal of Bacteriology 1998, 180:2027-2032.

30. Biegel E, Schmidt S, Gonzalez JM, Muller V: Biochemistry, evolution and physiological function of the Rnf complex, a novel ion-motive electron transport complex in prokaryotes. Cell Mol Life Sci 2011, 68:613-634.

31. Saum R, Schlegel K, Meyer B, Muller V: The F1FO ATP synthase genes in Methanosarcina acetivorans are dispensable for growth and ATP synthesis. FEMS Microbiology Letters 2009, 300(2):230-236.

32. Simianu M, Murakami E, Brewer JM, Ragsdale SW: Purification and properties of the heme- and iron-sulfur- containing heterodisulfide reductase from Methanosarcina thermophila. Biochemistry 1998, 37(28):10027-10039.

33. Carbon-dependent control of electron transfer and central carbon pathway genes for methane biosynthesis in the Archaean, Methanosarcina acetivorans strain C2A.

34. Lessner DJ, Li L, Li Q, Rejtar T, Andreev VP, Reichlen M, Hill K, Moran JJ, Karger BL, Ferry JG: An unconventional pathway for reduction of $\mathrm{CO} 2$ to methane in CO-grown Methanosarcina acetivorans revealed by proteomics. Proc Natl Acad Sci USA 2006, 103:17921-17926.

35. Rother M, Oelgeschlager E, Metcalf WM: Genetic and proteomic analyses of $\mathrm{CO}$ utilization by Methanosarcina acetivorans. Arch Microbiol 2007, 188(5):463-472.

36. Rother M, Metcalf WW: Anaerobic growth of Methanosarcina acetivorans C2A on carbon monoxide: an unusual way of life for a methanogenic archaeon. Proc Natl Acad Sci USA 2004, 101:16929-16934.

37. Zinder SH, Mah RA: Isolation and characterization of a thermophilic strain of Methanosarcina unable to use $\mathrm{H} 2-\mathrm{CO} 2$ for methanogenesis. Applied and Environmental Microbiology 1979, 38:996-1008.

38. Zinder SH, Sowers KR, Ferry JG: Methanosarcina thermophila sp. nov., a thermophilic, acetotrophic, methane-producing bacterium. Int I Syst Bacteriol 1985, 35:522-523.

39. Li Q, Li L, Rejtar T, Lessner DJ, Karger BL, Ferry JG: Electron transport in the pathway of acetate conversion to methane in the marine archaeon Methanosarcina acetivorans. Journal of Bacteriology 2006, 188:702-710.

40. Sowers KR, Baron SF, Ferry JG: Methanosarcina acetivorans sp. nov., an acetotrophic methane-producing bacterium isolated from marine sediments. Applied and Environmental Microbiology 1984, 47:971-978.

41. Sowers KR, Nelson MJK, Ferry JG: Growth of acetotrophic, methaneproducing bacteria in a pH auxostat. Curr Microbiol 1984, 11:227-230

42. Terlesky KC, Nelson MJK, Ferry JG: Isolation of an enzyme complex with carbon monoxide dehydrogenase activity containing a corrinoid and nickel from acetate-grown Methanosarcina thermophila. Journal of Bacteriology 1986, 168:1053-1058.

43. Kalb VF, Bernlohr RW: A new spectrophotometric assay for protein in cell extracts. Anal Biochem 1977, 82:362-371.

44. Graves MC, Mullenbach GT, Rabinowitz JC: Cloning and nucleotide sequence determination of the Clostridium pasteurianum ferredoxin gene. Proc Natl Acad Sci 1985, 82:1653-1657.

45. Tamura K, Dudley J, Nei M, Kumar S: MEGA4: Molecular evolutionary genetics analysis (MEGA) software version 4.0. Mol Biol Evol 2007, 24(8):1596-1599.

46. Larkin MA, Blackshields G, Brown NP, Chenna R, McGettigan PA, McWilliam H, Valentin F, Wallace IM, Wilm A, Lopez R, et al: Clustal W and Clustal X version 2.0. Bioinformatics 2007, 23(21):2947-2948.

doi:10.1186/1471-2180-11-165

Cite this article as: Wang et al:: Electron transport in acetate-grown Methanosarcina acetivorans. BMC Microbiology 2011 11:165. 